\title{
A bondgraph approach to formation control using relative state measurements
}

\author{
Geoff Stacey, Robert Mahony, Peter Corke
}

\begin{abstract}
In this paper we apply port-Hamiltonian theory with the bondgraph modelling approach to the problem of formation control using partial measurements of relative positions. We present a control design that drives a group of vehicles to a desired formation without requiring inter-vehicle communications or global position and velocity measurements to be available. Our generic approach is applicable to any form of relative measurement between vehicles, but we specifically consider the important cases of relative bearings and relative distances. In the case of bearings, our theory closely relates to the field of image-based visual servo (IBVS) control. We present simulation results to support the developed theory.
\end{abstract}

\section{INTRODUCTION}

Recent years have seen a growing interest in the application of formation control theory to unmanned vehicles. Some of the tasks motivating formation control include exploration using sensor networks [6], spacecraft interferometry [2] and the movement or manipulation of objects [8].

In the formation control literature, it is commonly assumed that each vehicle is able to obtain measurements of its own state, such as pose, linear velocity and angular velocity with respect to the inertial frame. A significant body of the literature focuses on how to achieve a decentralised control design that is scalable to large formations, and how the necessary inter-vehicle communications should be handled in the presence of delays or time-varying topologies [12], [18], [19]. There are a number of common approaches for the problem of formation control, including a leader-follower based design [6], [13], virtual structure control [7], [17], and behavioural control [1], [10]. Passivity theory has also proved to be a valuable tool in designing stable formation control algorithms [10], [12]. Formation control using an artificial potential based on relative distances has been considered by Leonard and Fiorelli [15].

In the case of aerial vehicles, full state measurements, and in particular measurements of position and linear velocity, can be difficult to obtain. They require external infrastructure such as motion-capture systems, high-precision differential GPS, fast and high powered vision systems, or highly sophisticated estimators that depend on accurate dynamic models of the vehicles. When we consider the problem of formation control, it can be more appropriate to work with relative state measurements (i.e. the position or velocity of one vehicle with respect to another). Recent experimental

G. Stacey and R. Mahony are with the Research School of Information Science and Engineering, Australian National University, ACT 0200, Australia. \{geoffrey.stacey, robert.mahony\}@anu.edu.au

P. Corke is with the CyPhy Laboratory, School of Electrical Engineering and Computer Science, Queensland University of Technology, QLD 4000, Australia. peter.corke@qut.edu.au results for large formations of quadrotors using full relative state measurements are given by Turpin et al. [20], [21] and Franchi et al. [10].

Partial measurements of relative state are often available even when the vehicles are unable to communicate their global state. An example would be when the vehicles are able to identify each other in video data obtained from onboard cameras, but there is no inter-vehicle communication. The control of motion based on visual information is known as image-based visual servo (IBVS) control and has been extensively studied in robotics over the last twenty years (see the tutorials [4], [5]). Passivity based control concepts have been used to extend IBVS control to fully actuated dynamical systems [11]. Mahony et al. [16] did similar work using bondgraph modelling and the port-Hamiltonian systems framework. This approach was shown to provide valuable insights for the design and stability analysis of the control law. There has been limited prior work regarding the use of vision to control vehicle formations (see e.g. [6], [13]). More recently, Franchi et al. [9] used relative bearings from vision for formation control. This work implemented a constructive nonlinear control design that was dependent on the specific properties of the bearing measurements and agent configuration.

In this paper, we apply the principles of port-Hamiltonian theory and bondgraph modelling to the problem of controlling a formation of aerial robotic vehicles based on partial relative state measurements. Unlike many designs for formation control, we do not assume that the vehicles are able to actively communicate with each other. The approach taken assumes that each vehicle can sense its neighbouring vehicles, providing partial relative state measurements such as visual bearings or range. The key contribution of the paper is to provide a natural modelling framework in which virtual mechanical coupling between agents in a network can be modelled and designed in a simple and intuitive manner. The energy continuous nature of the port-Hamiltonian model leads to a simple and intuitive stability analysis, and we prove asymptotic stability of a desired formation for a network under simple and generic conditions. Although we are particularly motivated by bearing only measurements such as those provided by a camera, we develop a general control architecture that can be applied to a variety of relative state measurements including bearing, range, full relative position, or any other partial relative state measurement. We provide simulation results to demonstrate a typical response of the proposed control architecture for a formation stabilisation problem with a network graph where the measurements are relative bearings and ranges. 
After the present Introduction, we provide a formal statement of the problem in Section II. In Section III, we develop a bondgraph model for the formation control of aerial vehicles, using generic relative measurements as might be obtained from onboard cameras or time-of-flight sensors. An analysis of the resulting design is provided in Section IV. We then present simulation results in Section $\mathrm{V}$ to support the theory, and in Section VI we provide a short conclusion.

\section{Problem Formulation}

Consider the scenario of $n$ free-moving vehicles, with the $i$ th vehicle having a mass $m_{i}$ and a body-fixed frame $\left\{B_{i}\right\}$. We denote the vector $p_{i} \in \mathbb{R}^{3}$ and rotation matrix $R_{i} \in$ $S O(3)$ to be the position and orientation, respectively, of the $i$ th vehicle expressed in the inertial reference frame $\{A\}$.

For this paper, we are concerned with controlling the relative positions of the vehicles. The attitude dynamics are assumed to be controlled and full state estimates of the vehicle attitude are assumed to be available as is standard in the literature [2], [12]. The dynamics of the $i$ th vehicle with control input $F_{i} \in \mathbb{R}^{3}$ (expressed in the inertial frame) are described using the point mass model [8], [16],

$$
F_{i}-\frac{\partial U_{i}}{\partial p_{i}}\left(p_{i}\right)=m_{i} \ddot{p}_{i}
$$

where, in our case, the potential energy $U_{i}\left(p_{i}\right)$ is solely due to gravity and is given by,

$$
U_{i}:=m_{i} g p_{i}^{z} \text {. }
$$

Here, $g$ is the acceleration due to gravity and it is assumed that the $z$-component $p_{i}^{z}$ of $p_{i}$ is zero at ground level with the $z$-axis of the inertial frame pointing upwards. The mechanical Hamiltonian $H_{i}^{\text {mech }}\left(p_{i}, \dot{p}_{i}\right)$ for the $i$ th vehicle is given by,

$$
H_{i}^{\text {mech }}\left(p_{i}, \dot{p}_{i}\right):=T_{i}\left(\dot{p}_{i}\right)+U_{i}\left(p_{i}\right),
$$

where $T_{i}:=\frac{1}{2 m_{i}}\left\|m_{i} \dot{p}_{i}\right\|^{2}$ is the kinetic energy due to the linear velocity of the vehicle. We have used $\|\cdot\|$ to denote the Euclidean norm of a vector.

The structure of a formation control problem is commonly represented as a network graph, as in Figure 1a [12], [18]. We denote the undirected connected network graph $\mathcal{G}:=$ $(\mathcal{V}, \mathcal{E})$, where $\mathcal{V}$ is the set of $n$ nodes in the graph (each representing a vehicle in our case) and $\mathcal{E}$ is the set of $m$ edges (representing links associated with the relative variables between vehicles). A neighbour of vehicle $i$ is a vehicle that is connected to it by an edge, and $\mathcal{N}_{i} \subseteq \mathcal{V}$ is the set of nodes corresponding to all neighbours of vehicle $i$. The set of edges connected to vehicle $i$ is written $\mathcal{E}_{i} \subseteq \mathcal{E}$.

We are interested in the use of relative state measurements that describe the position and velocity of one vehicle with respect to another. We shall assume that the measurements are exact and that each vehicle de-rotates the relative state measurements from the body-fixed frame to the inertial reference frame, based on its local attitude estimate.

Define the relative position associated with a link $k$ as $q_{k}:=p_{i}-p_{j}$, where vehicles $i$ and $j>i$ are those connected by the link. Let

$$
\dot{q}_{k}=\dot{p}_{i}-\dot{p}_{j}
$$

represent the relative velocity of vehicle $i$ with respect to vehicle $j$.

Partial relative position measurements are measurements that only observe some of the dimensions of $q_{k}$. The two most important examples are relative distances or ranges (as might be measured by a time-of-flight sensor) and relative bearings (as would be obtained by an onboard camera). For a generic (possibly partial) measurement of relative position we will use the notation $y_{k}:=y_{k}\left(q_{k}\right)$. The set of possible values for a given type of measurement shall be referred to as the sensor space.

The measurement Jacobian is the differential of the relative measurement with respect to the relative state,

$$
N_{k}:=\frac{\partial y_{k}}{\partial q_{k}} \text {. }
$$

The relationship between a vehicle's relative velocity and the perceived velocity in an arbitrary sensor space is,

$$
\dot{y}_{k}=N_{k} \dot{q}_{k} \text {. }
$$

When all three degrees of freedom of $q_{k}$ are observed (i.e. $y_{k}=q_{k}$ ), the measurement Jacobian is simply the $3 \times 3$ identity matrix $\left(N_{k}=I_{3}\right)$.

Let $r_{k}:=\left\|q_{k}\right\|$ denote the relative (Euclidean) distance between vehicles $i$ and $j$. The time differential of this distance is,

$$
\dot{r}_{k}=\frac{d}{d t}\left(\sqrt{q_{k}^{\top} q_{k}}\right)=2 \times \frac{1}{2} \frac{q_{k}^{\top} \dot{q}_{k}}{\sqrt{q_{k}^{\top} q_{k}}}=\frac{q_{k}^{\top}}{r_{k}} \dot{q}_{k} .
$$

and the associated distance Jacobian is $M_{k}:=\frac{q_{k}^{\top}}{r_{k}} \in \mathbb{R}^{1 \times 3}$.

Let the bearing $s:=(x, y, z)^{\top} \in \mathbb{S}^{2}$ be represented as unit vector in Cartesian space, i.e. $\mathbb{S}^{2}:=\left\{(x, y, z)^{\top}: x, y, z \in\right.$ $\left.\mathbb{R}, x^{2}+y^{2}+z^{2}=1\right\}$. Measurements in this sensor space can be modelled by projecting a point $q_{k} \in \mathbb{R}^{3}$ to the point,

$$
s_{k}:=\frac{1}{r_{k}} q_{k} \in \mathbb{S}^{2} .
$$

The associated measurement Jacobian can be found by taking the time-derivative of this:

$$
\begin{aligned}
\dot{s}_{k} & =\frac{\dot{q}_{k}}{r_{k}}-\frac{\dot{r}_{k}}{r_{k}} s_{k} \\
& =\frac{1}{r_{k}}\left(I_{3}-s_{k} s_{k}^{\top}\right) \dot{q}_{k} .
\end{aligned}
$$

Here, $I_{3}$ is the $3 \times 3$ identity matrix and the last line follows from (7). The image Jacobian $L_{k}$ is,

$$
L_{k}:=\frac{1}{r_{k}}\left(I_{3}-s_{k} s_{k}^{\top}\right) \in \mathbb{R}^{3 \times 3} .
$$

The idea of an image Jacobian is well established in classical IBVS theory, and it shall be used for the same purpose in this paper. In all cases we have expressed the measurement Jacobians in the inertial frame of reference.

The objective of the control design is to ensure that each measurement $y_{k}$ converges to a desired constant value $y_{k}^{*}$. These vectors define the goal formation for the vehicles, as parameterised by the sensor measurements. Equivalently, we may say that we require the errors $\tilde{y}_{k}:=y_{k}-y_{k}^{*}$ to converge to zero. 


\section{BONDGRAPH MOdEl OF FORMATION CONTROL}

In this section we develop a bondgraph model for the problem of formation control with relative measurements between vehicles. We begin by presenting a model of a single vehicle, and then proceed by showing how a link between two vehicles can be modelled to represent the measurements and energy exchanges between them. For an introduction to bondgraph modelling, we refer the reader to [3].

In Figure 1, we show two different ways of representing the formation control scenario. On the left we have a network graph that uses the classical notation, where a circle (vertex) represents a vehicle and a two-way arrow (edge) is used to indicate that information can flow between two vehicles.

The graph in Figure $1 b$ is a new notation that we propose in this paper. It contains the additional information necessary to serve as a shorthand version of the bondgraph model that we will use to model the virtual mechanical coupling between the vehicles. In Figure 1b, the edges represent an exchange and storage of virtual energy between two vehicles, with energy flow or power being considered positive in the direction indicated by the half-arrow. It is convenient to think of the edges in Figure 1b as virtual springs that interconnect the two vehicles, although it is possible to model an arbitrary virtual mechanical coupling using the same approach. Each edge is labelled with an index $k$ and is associated with a generalised force $\epsilon_{k}$. It is straightforward to see that if the two vehicles are interconnected by a spring then the force applied to one vehicle is equal and opposite to the force applied to the other vehicle. We show later that this is also the case for more sophisticated virtual couplings. The direction of the arrow of the edge indicates which vehicle takes $\epsilon_{k}$ as positive while the other vehicle takes $\epsilon_{k}$ as negative (the definition for $q_{k}$ in Section II assumes that the arrow goes from vehicle $j$ to vehicle $i<j$ ). Note that we may have more than one link between two vehicles if those links use different sensor modalities. The perpendicular strokes at either end of each link are called causal strokes in bondgraph modelling, and here they indicate that both vehicles have force applied by the virtual coupling. Although the causal strokes are not strictly necessary in the shorthand notation that we use, they are convenient to include since they make it clear that the bond is bi-directional.

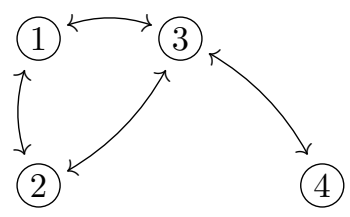

(a) Classical network graph representation of a formation control scenario.

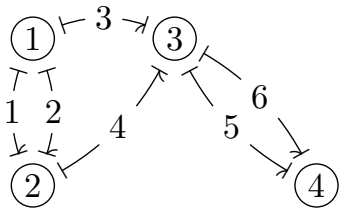

(b) Proposed representation of a formation control scenario. This includes additional information relevant to the bondgraph model.

Fig. 1

We model the physical dynamics of vehicle $i$ by the bondgraph in Figure 2. We use the notation $\sum_{i}^{\text {mech }}$ to represent the mechanical subsystem of vehicle $i$, whose dynamics are given by (1). The line passing from the mechanical subsystem to the modulated transformer (MTF) represents a bond through which energy can be transferred into or out of the vehicle dynamics. The power flowing through this bond is the contraction of the effort, ${ }^{i} F_{i}$, with the flow, ${ }^{i} \dot{p}_{i}$ (the top-left index means that these variables are expressed in the body-fixed frame $\left.\left\{B_{i}\right\}\right)$. The half-arrow indicates the direction in which power is measured positive. Lastly, the causal stroke is placed on the side of the mechanical subsystem to indicate that the system's velocity is causally dependent on the applied force.

$$
\sum_{i}^{\text {mech }} h^{{ }^{i} F_{i}}{ }_{{ }^{i} \dot{p}_{i}} \mathrm{MTF}
$$

Fig. 2: Model of an energy bond through which power can be provided to the mechanical system of vehicle $i$.

The structure associated with the local control of a single vehicle $i$ is presented in Figure 3. The MTF acts to de-rotate the force ${ }^{i} F_{i}:=R_{i}^{\top} F_{i}$ and velocity ${ }^{i} \dot{p}_{i}:=R_{i}^{\top} \dot{p}_{i}$ into the inertial reference frame. The $R_{i}$ at the base of the normal arrow going into the MTF indicates that the rotation matrix is required for the transform function.

To the right of the MTF is a 1-junction [3]. A 1-junction represents a parallel connection of virtual mechanical components. In this case these are a spring like potential $C_{i}^{\text {comp }}$, a damper $D_{i}$, and the link to the virtual couplings. The virtual force $\tau_{i}$ on the rightmost branch derives from the virtual couplings with other vehicles based on the relative sensor measurements available. The forces from each of the components are summed at the 1 -junction to obtain $F_{i}$.

The virtual potential $C_{i}^{\text {comp }}$ is used to compensate for gravity. We define an associated Hamiltonian $H_{i}^{\text {comp }}\left(p_{i}\right):=$ $G_{i}-U\left(p_{i}\right)$ that counteracts the gravitational potential, where $G_{i}$ is a large positive constant such that $H_{i}^{\text {comp }}$ is positive in the domain in which the system will maneouvre. The compensating force is computed as $\frac{\partial H_{i}^{\text {comp }}}{\partial p_{i}}\left(p_{i}\right)$.

Damping is introduced by dissipating virtual energy through the upper branch of the 1-junction. This acts as a friction force $\delta_{i}:=D_{i} \dot{p}_{i}$ in the system dynamics, where $D_{i}>0$ is a positive definite gain matrix.

We use the notation $\sum_{i}^{\text {vehicle }}$ to represent an individual vehicle along with its local controller, as shown on the righthand side of Figure 3. Note that we leave the rightmost 1-junction in Figure 3 external to the system block, providing a node to which the virtual couplings can be attached.

The next stage in the development is to model the virtual mechanical couplings that we introduce between vehicles. In this paper we consider the simplest of mechanical couplings - a virtual spring potential - implemented with the relative sensor modality $y_{k}$ of the vehicles (i.e. of one of the links in Figure 1b). Each link between a pair of vehicles will correspond to a particular sensor measurement, and will connect the rightmost 1 -junction in the model for vehicle $i$ (Figure 3) to that of vehicle $j$. The model for a link between vehicles is shown in Figure 4. 


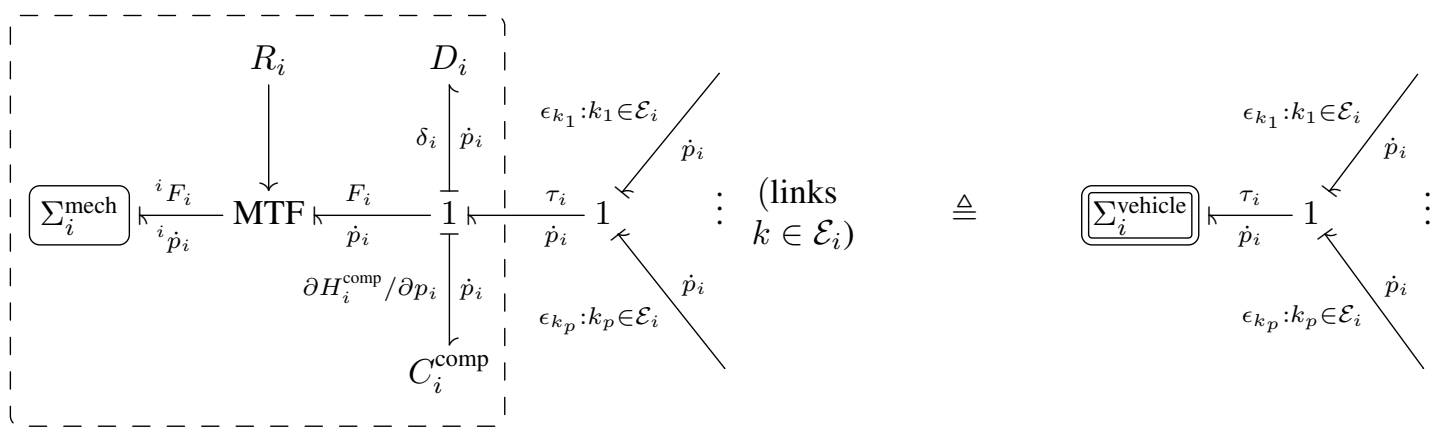

Fig. 3: Bondgraph model of a single vehicle in the formation control problem. Each of the rightmost bonds will connect to a link between this vehicle and another. This vehicle's subsystem will later be denoted as indicated on the right.

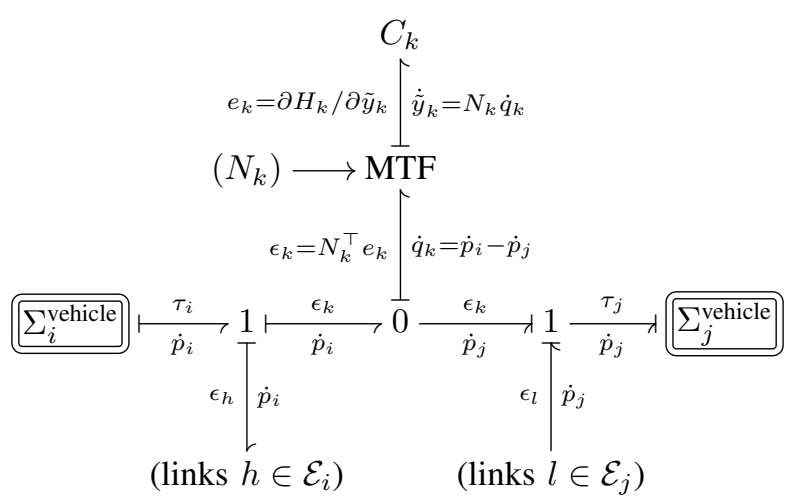

Fig. 4: Bondgraph model of a control link between two vehicles, given a relative measurement $y_{k}$. Note the difference in the relative orientation of the energy bonds going to each vehicle's subsystem. The relative orientations of the links $h$ and $l$ may vary.

The relative velocity (4) is a sum of flows and can therefore be represented in the bondgraph by the use of a 0 junction [3] as shown in Figure 4. The 0-junction represents a serial interconnection of mechanical elements; in this case it shows that vehicle $i$ is connected to the virtual coupling, which is connected in turn to vehicle $j$. The forces $\epsilon_{k}$ in all bonds attached to a 0 -junction are equal, as is natural for mechanical elements connected in series. This is the case for any mechanical coupling and justifies the notation introduced in Figure 1b. From the relative directions of the half-arrows, we can see that this link will apply equal and opposite virtual forces to the two vehicles. The causality strokes are placed to show that the force $\epsilon_{k}$ applied to each vehicle's subsystem will determine the flows $\dot{p}_{i}$ and $\dot{p}_{j}$. The velocities at a zero junction sum, so (4) indicates the relative compression or extension of the interconnection.

In order to implement the virtual coupling, we require the relative sensor measurement. The measurement Jacobian relationships developed in Section II are used to define an MTF that transforms the relative velocity $\dot{q}_{k}$ into the relative sensor flow, as given in (6), for $k \in \mathcal{E}_{i} \cap \mathcal{E}_{j}$. We choose to make $\dot{\tilde{y}}_{k}=\dot{y}_{k}$ the flow variable for simplicity, because it is convenient to define the energy in the storage element as a function of $\tilde{y}_{k}$. The MTF induces a natural adjoint mapping on the sensor effort,

$$
\epsilon_{k}:=N_{k}^{\top} e_{k},
$$

where $e_{k}$ is the effort in the sensor space.

The measurement Hamiltonian that defines the energy in the storage element is given by the function,

$$
H_{k}^{\text {meas }}\left(\tilde{y}_{k}\right):=\frac{1}{2} \tilde{y}_{k}^{\top} K_{k} \tilde{y}_{k},
$$

where $K_{k}$ is a positive definite gain constant of appropriate dimensions. The goal vectors $y_{k}^{*}$ are assumed to be constant.

The effort $e_{k}$ in the sensor space is given by,

$$
e_{k}:=\frac{\partial H_{k}}{\partial \tilde{y}_{k}}=K_{k} \tilde{y}_{k} .
$$

The effort $e_{k}$ in the sensor space is transformed into $\epsilon_{k} \in \mathbb{R}^{3}$ by the adjoint operation $N_{k}^{*}=N_{k}^{\top}$ of the modulated transformer function; it is straightforward to verify that here the power $\left\langle e_{k} \mid \dot{\tilde{y}}_{k}\right\rangle$ into the modulated transformer equals the power $\left\langle\epsilon_{k} \mid \dot{q}_{k}\right\rangle$ out. The resulting virtual force $\epsilon_{k}$ is then passed to the 1-junction associated with each of the interconnected vehicle subsystems.

\section{ANALYSIS OF NETWORK STABILITY}

The virtual couplings introduced in Section III lead to dynamic evolution of the formation of vehicles. In this section we analyse the closed-loop behaviour of the formation and show that for almost all initial conditions the system converges to the desired equilibrium.

In order to analyse the network dynamics, we introduce notation to refer to stacked variables. Define,

$$
p:=\left(\begin{array}{c}
p_{1} \\
\vdots \\
p_{n}
\end{array}\right) \quad \tau:=\left(\begin{array}{c}
\tau_{1} \\
\vdots \\
\tau_{n}
\end{array}\right) \quad y:=\left(\begin{array}{c}
y_{1} \\
\vdots \\
y_{m}
\end{array}\right) \quad y^{*}:=\left(\begin{array}{c}
y_{1}^{*} \\
\vdots \\
y_{m}^{*}
\end{array}\right)
$$

and the block-diagonal matrices,

$$
D:=\operatorname{diag}\left\{D_{1}, \ldots, D_{n}\right\} \quad K:=\operatorname{diag}\left\{K_{1}, \ldots, K_{m}\right\} .
$$

The total energy $H$ of the system is,

$$
\begin{aligned}
H & :=\sum_{i=1}^{n}\left(H_{i}^{\text {mech }}+H_{i}^{\text {comp }}\right)+\sum_{k=1}^{m} H_{k}^{\text {meas }} \\
& =\sum_{i=1}^{n}\left(\frac{1}{2 m_{i}}\left\|m_{i} \dot{p}_{i}\right\|^{2}+G_{i}\right)+\frac{1}{2} \tilde{y}^{\top} K \tilde{y} .
\end{aligned}
$$


By construction, and since all interconnections have been made in an energy continuous manner, the total energy dissipated from the system is given by,

$$
\frac{d}{d t} H(p, \dot{p})=-\dot{p}^{\top} D \dot{p}
$$

We now present the following theorem for the formation control problem discussed.

Theorem 4.1: Consider a group of $n$ vehicles, with the dynamics of the $i$ th vehicle being described by (1). Suppose that there exist $m$ links, each of which is associated with a relative position measurement $y_{k}$ between a pair of vehicles $i$ and $j$, as indicated by the bondgraph in Figure 4. Assume that the relevant measurement Jacobians $N_{k}$ are known. Assume that the total energy has a unique global minimum. Then, for almost all initial conditions $(p(0), \dot{p}(0))$ the closed-loop trajectory $(p(t), \dot{p}(t)) \rightarrow\left(p^{*} \in \mathcal{F}, 0\right)$, where $\mathcal{F} \in \mathbb{R}^{3 \times n}$ is the set of points satisfying $y=y^{*}$.

Proof: Since the total energy in the system is bounded by its initial value, the maximum velocity of any vehicle is bounded for all time. It follows that the trajectories of the network exist for all time. Applying Lyapunov's theorem and LaSalle's principle, it follows that $(p, \dot{p})$ converges to the largest forward invariant set $\mathcal{X}$ such that $\dot{p}^{\top} D \dot{p}=0$ and hence that $\dot{p} \rightarrow 0$. On LaSalle's invariant set, and recalling (1), the fact that $\dot{p} \equiv 0$ implies that $\tau=0$.

The kinetic energy of the Hamiltonian is positive definite in the generalised velocity $\dot{p}(t)$ and it follows that the critical points, and in particular the unique minima, of the Hamiltonian correspond to critical points of the associated total potential energy of the network. It follows that the set characterised by $\dot{p}=0$ and $\tau=0$ corresponds to the critical points of the total potential energy of the network. By assumption there is a unique global minimum of the Hamiltonian, and using classical ODE theory [14], the system solution will converge to this for almost all initial conditions. The exceptional set will consist of saddle points and local maxima of the total potential energy of the network that have basins of attraction of zero measure.

In Theorem 4.1, the assumption that the Hamiltonian has a unique global minimum can be relaxed to existence of a local minima if one is willing to work within a basin of attraction for convergence of the closed-loop system. In practice, and especially with bearing measurements, it is more common to work with a local analysis [4].

A further point of interest is that if only the relative formation need be stabilised, then the virtual potential in the Hamiltonian will be invariant under a rigid-body translation of the whole formation. Formally, the Hamiltonian will no longer have a local minima. Given that the dissipation $D_{i}$ is applied directly to individual agents it is straightforward to extend the above proof to show that we converge to some configuration for which the Hamiltonian is minimised. A more interesting situation occurs when the relative measurements themselves are insufficient to fully determine a unique relative structure of the formation. For example, if all relative measurements are bearings only then the scale of the formation is unobservable and the local minima of the Hamiltonian will be invariant to total scale of the formation. The same idea mentioned for the invariance of the Hamiltonian with respect to translation can be used to analyse this situation. Full analysis of these special cases are beyond the scope of the present paper, although we do note the invariance to translation in the simulation.

\section{Simulations and Discussion}

In this section we present simulation results to support the proposed approach. The case considered consists of a typical configuration with a goal position $p^{*}$ that is fully defined up to a rigid-body translation (the formation is not free to rotate because the desired relative bearings are defined in the inertial frame).

The results for the simulation are shown in Figure 5. We have four vehicles with a network topology as depicted in Figure 1b. Here, edges 1, 3, and 5 use measurements of relative distance and edges 2, 4, and 6 are associated with measurements of relative bearing. To verify that the goal formation is fully defined, we note that the position of vehicle 2 with respect to vehicle 1 is fixed by the associated measurements of relative distance and bearing (edges 1 and 2 , respectively), and that the same applies for vehicles 3 and 4. The relative bearing of vehicle 3 with respect to vehicle 2 constrains its goal position to a straight line originating from vehicle 2, and we have chosen the desired relative distance of vehicle 3 with respect to vehicle 1 such that only one point along the line can satisfy this constraint. Therefore, the goal formation is fully specified and corresponds to a global minimum in the total energy $H$ of the system.

The trajectories of each vehicle are plotted in Figure 5a. In Figure 5b, we plot the sum squared error of all relative bearings and distances for each vehicle. Note that this sum includes, for example, the error in the relative bearing of vehicle 4 with respect to vehicle 1 (defined by the goal formation) even though this measurement is not used for the control of the vehicles. This plot confirms that all relative bearings and distances converge to the desired value, and hence that $p \rightarrow p^{*} \in \mathcal{F}$. The total energy of the measurement Hamiltonians, and the total energy $H$ of the system, are both plotted in Figure $5 \mathrm{c}$. We note that the total energy in the system is always decreasing, while the energy in the measurement Hamiltonians is sometimes increasing. This is due to a low damping term that enables the vehicles to overshoot the desired position, thus transferring mechanical energy back into the measurement Hamiltonians. The plot of the total energy suggests an exponential rate of convergence.

\section{CONCLUSION}

In this paper we considered the problem of controlling a formation of vehicles using partial measurements of relative position. Our controller was based on introducing virtual mechanical coupling between agents in the network. The bondgraph modelling technique and port-Hamiltonian system framework were used to develop the control and analyse the closed-loop response. The approach is generic enough to be applicable to any type of relative position measurement, and we demonstrated this for the particular cases of relative distances and bearings. 


\section{ACKNOWLEDGEMENT}

This research was supported by the Australian Research Council through Future Fellowship FT0991771 "Foundations of Vision Based Control of Robotic Vehicles".

\section{REFERENCES}

[1] T. Balch and R. C. Arkin. Behavior-based formation control for multirobot teams. IEEE Transactions on Robotics and Automation, 14(6):926-939, December 1998.

[2] R. W. Beard, J. Lawton, and F. Y. Hadaegh. A coordination architecture for spacecraft formation control. IEEE Transactions on Control Systems Technology, 9(6):777-790, November 2001.

[3] W. Borutzky. Bond graph modelling and simulation of mechatronic systems an introduction into the methodology. In European Conference on Modelling and Simulation, May 2006.

[4] F. Chaumette and S. Hutchinson. Visual servo control. I: basic approaches. IEEE Robotics \& Automation Magazine, 13(4):82-90, December 2006.

[5] F. Chaumette and S. Hutchinson. Visual servo control. II: advanced approaches. IEEE Robotics \& Automation Magazine, 14(1):109-118, March 2007.

[6] A. K. Das, R. Fierro, V. Kumar, J. P. Ostrowski, J. Spletzer, and C. J. Taylor. A vision-based formation control framework. IEEE Transactions on Robotics and Automation, 18(5):813-825, October 2002.

[7] M. Egerstedt and X. Hu. Formation constrained multi-agent control. IEEE Transactions on Robotics and Automation, 17(6):947-951, December 2001.

[8] J. Fink, N. Michael, S. Kim, and V. Kumar. Planning and control for cooperative manipulation and transportation with aerial robots. International Journal of Robotics Research, 30(3):324-334, March 2011.

[9] A. Franchi, C. Masone, V. Grabe, M. Ryll, H. H. Bülthoff, and P. Robuffo Giordano. Modeling and control of UAV bearing formations with bilateral high-level steering. International Journal of Robotics Research, 31(12):1504-1525, November 2012.

[10] A. Franchi, C. Secchi, H. I. Son, H. H. Bülthoff, and P. Robuffo Giordano. Bilateral teleoperation of groups of mobile robots with time-varying topology. IEEE Transactions on Robotics, 28(5):10191033, October 2012.

[11] M. Fujita, H. Kawai, and M. W. Spong. Passivity-based dynamic visual feedback control for three-dimensional target tracking: stability and $L_{2}$-gain performance analysis. IEEE Transactions on Control Systems Technology, 15(1):40-52, January 2007.

[12] T. Hatanaka, Y. Igarashi, M. Fujita, and M. W. Spong. Passivity-based pose synchronization in the three dimensions. IEEE Transactions on Automatic Control, 57(2):360-375, February 2012.

[13] E. N. Johnson, A. J. Calise, R. Sattigeri, Y. Watanabe, and V. Madyastha. Approaches to vision-based formation control. In IEEE Conference on Decision and Control, December 2004.

[14] H. K. Khalil. Nonlinear Systems. Prentice Hall, 3rd edition, 2002.

[15] N. E. Leonard and E. Fiorelli. Virtual leaders, artificial potentials and coordinated control of groups. In IEEE Conference on Decision and Control, December 2001.

[16] R. E. Mahony and S. Stramigioli. A port-Hamiltonian approach to image-based visual servo control for dynamic systems. International Journal of Robotic Research, 31(11):1303-1319, September 2012.

[17] W. Ren and R. W. Beard. Decentralized scheme for spacecraft formation flying via the virtual structure approach. Journal of Guidance, Control and Dynamics, 27(1):73-82, January-February 2004.

[18] C. Secchi, A. Franchi, H. H. Bülthoff, and P. Robuffo Giordano. Bilateral teleoperation of a group of UAVs with communication delays and switching topology. In IEEE International Conference on Robotocis and Automation, May 2012.

[19] H. G. Tanner, A. Jadbabaie, and G. J. Pappas. Flocking in fixed and switching networks. IEEE Transactions and Automatic Control, 52(5):863-868, May 2007.

[20] M. Turpin, N. Michael, and V. Kumar. Decentralized formation control with variable shapes for aerial robots. In IEEE International Conference on Robotocs and Automation, May 2012.

[21] M. Turpin, N. Michael, and V. Kumar. Trajectory design and control for aggressive formation flight with quadrotors. Autonomous Robots, 33:143-156, February 2012.

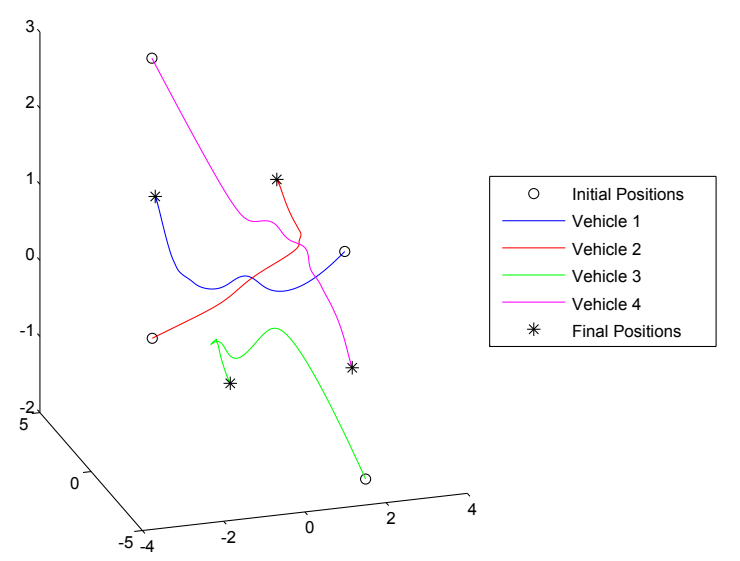

(a) The position trajectories for the simulation. The vehicles converge to a fully defined goal position $p^{*}$.
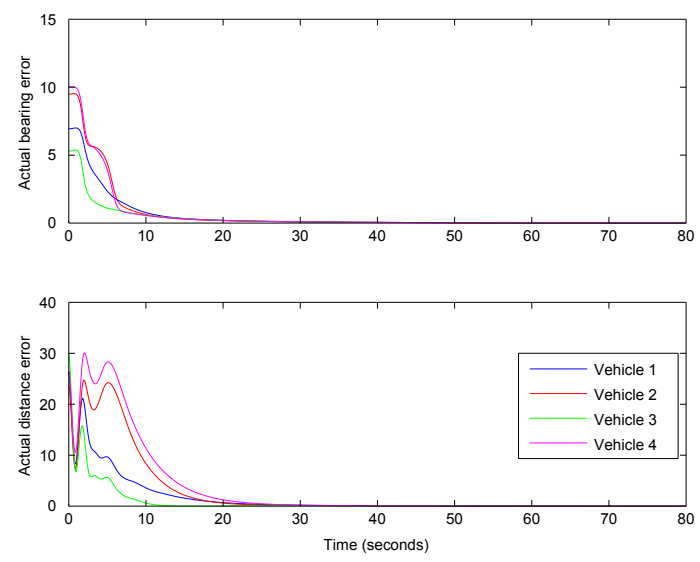

(b) The sum squared errors of all relative bearings and distances for each vehicle in the simulation. The relative measurements converge to the desired values.
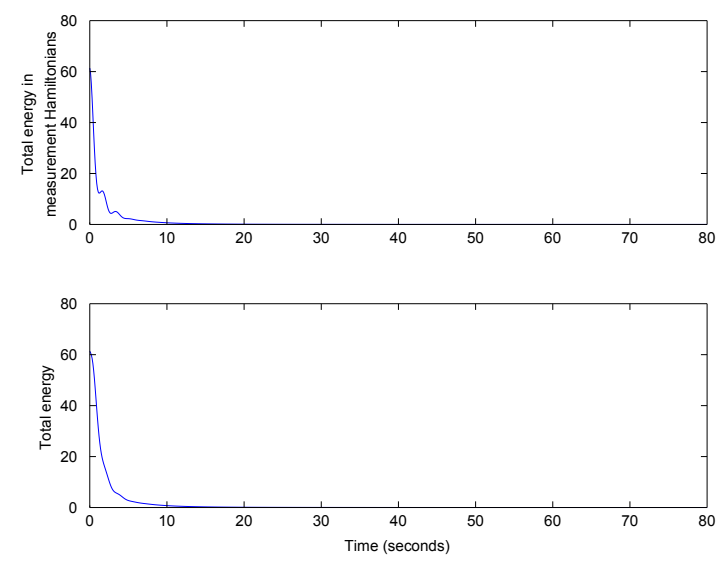

(c) Top: the energy in the measurement Hamiltonians. Bottom: the total energy in the system, exluding a constant value $\sum_{i=1}^{n} G_{i}$.

Fig. 5 